\title{
Classification of planetary nebulae by cluster analysis and artificial neural networks
}

\author{
M. Faúndez-Abans ${ }^{1}$, M.I. Ormeño ${ }^{2}$ and M. de Oliveira-Abans ${ }^{1}$ \\ 1 Laboratório Nacional de Astrofísica, Caixa Postal 21, CEP:37.500-000,Itajubá, MG, Brazil \\ 2 Departamento de Física, Universidad de Santiago de Chile, Casilla 307, Correo 2, Santiago, Chile
}

Received July 24; accepted September 18, 1995

\begin{abstract}
According to the chemical composition, a sample of 192 Planetary Nebulae of different types has been re-classified, and 41 others have been classified for the first time, by means of two methods not employed so far in this field: hierarchical cluster analysis and supervised artificial neural network. The cluster analysis reveals itself as a good first guess for grouping Planetary Nebulae, while an artificial neural network provides reliable automated classification of this kind of objects.
\end{abstract}

Key words: planetary nebulae: general — methods: miscellaneous

\section{Introduction}

In the seventies, Planetary Nebulae (PN) were classified according to their chemical, spatial, kinematical, and morphological properties into four types, namely, Types I, II, III, and IV (for details on the classification see Peimbert 1978; Peimbert \& Serrano 1980; Peimbert \& Torres-Peimbert 1983; Peimbert 1990).

These nebulae are interesting tracers of the chemical evolution of the Galaxy and other galaxies. The analysis of the chemical properties of PN is important because it determines the abundances in the progenitor star, as well as the individual enrichment by the progenitor's nucleosynthesis, which, in turn, depends on the star's mass. According to Peimbert's classification, most nebulae are probably of Type II, having abundances $\mathrm{He} / \mathrm{H}<0.125$, $\log (\mathrm{N} / \mathrm{O})<-0.3$, distance to the galactic plane $|z|<1$ $\mathrm{kpc}$, and peculiar radial velocity $|v|<60 \mathrm{~km} / \mathrm{s}$.

New efforts were made in the eighties to classify PN, and Type II has been subsequently divided into two subtypes, named IIa and IIb, according to their nitrogen relative to hydrogen abundance ratio (see Faúndez-Abans \& Maciel 1987a). The PN which belong to the population of the galactic bulge were classified as Type $\mathrm{V}$ objects (see Maciel 1989 for details). Also, an interesting classification of PN into three types has been presented by Amnuel et al. (1989), based on the values of the planetary and progenitor's masses; this has been altered recently to four types, by Amnuel (1993).

Send offprint requests to: M. Faúndez-Abans
The separation of PN in groups has been useful in the study of the presence of radial and vertical abundance gradients in the Galaxy. A detailed study of radial gradients from Type II PN was carried out by FaúndezAbans \& Maciel (1986, 1987b), who found clear oxygen gradients, besides other heavy elements quoted in those works, and a diagnostics of vertical gradient was made by Faúndez-Abans \& Maciel (1988 and references quoted therein). Recently, Maciel \& Köppen (1994) have made new determinations of abundance gradients of $\mathrm{O}, \mathrm{Ne}, \mathrm{S}$, and $\mathrm{Ar}$, elements which should not be manufactured by the progenitor.

In this work, we have used a homogeneous and upto-date PN abundance data set, to first subdivide them, according to their chemical composition, by means of a cluster analysis and then, with these results in hand, to train an artificial neural network (ANN from now on) for classification of more objects.

\section{Procedure}

\subsection{The data}

The data for our classification test have been extracted from the homogeneous compilation of abundances for galactic PN given by Köppen et al. (1991), Chiappini (1993), and Maciel \& Chiappini (1994, and references quoted in their Table 1), where Type V PN are excluded. This compilation gathers abundance fractions of $\mathrm{He}, \mathrm{O}, \mathrm{N}, \mathrm{S}, \mathrm{C}, \mathrm{Ne}, \mathrm{Ar}$, and $\mathrm{Cl}$ relative to $\mathrm{H}$. Nonetheless, as several PN lack information on $\mathrm{C}, \mathrm{Ne}$, and $\mathrm{Cl}$, these chemical elements have not been included 
in our present analysis. We have thus worked with $233 \mathrm{ob}-$ jects. We have first considered only $\mathrm{PN}$ with a complete set of $\mathrm{He} / \mathrm{H}, \mathrm{O} / \mathrm{H}$, and $\mathrm{N} / \mathrm{H}$ ratios, and then $\mathrm{S} / \mathrm{H}$ and $\mathrm{Ar} / \mathrm{H}$ data, when available (see Sect. 3). Thirdly, we have included in the cluster analysis objects lacking information on He abundance.

\subsection{The cluster analysis}

The techniques of cluster and discriminant analysis are suitable for identifying and organizing those PN parameters of interest to arrange these objects in groups (these techniques are fully discussed in Anderberg 1973; Tatsuoka 1971; Johnson \& Wichern 1986).

The basics of the cluster analysis is to choose a measure of distance between the various families of parameters, from now on referred to as observations, and use it to decide whether an object belongs to a certain family. In this one-dimensional context, the method proceeds as follows: a) one sorts the observations in ascending order and treats each observation as a group with one member, b) one examines all pairs of adjacent groups to find those two which are closest, the distance between them being the distance to their nearest member, c) step "b" is repeated at will or until there is only one group. This simple method is capable of identifying the largest groups in a sequence of parameters (see Kendall \& Stuart 1968; Anderberg 1973; Marriolt 1974).

In this work we have used a more sophisticated algorithm, namely, the Wards's hierarchical cluster method (Ward \& Hook 1963; Ward 1963; Wishart 1969). This technique proceeds as follows: a) one starts with $n$ groups, each group consisting of one observation, i.e., a planetary; b) at each step the number of groups is reduced by one, through merging those two groups whose combination gives the least possible increase in distance " $w$ ", defined as follows:

$$
w=\sum_{i=1}^{g} \sum_{j=1}^{n_{i}}\left(x_{i j}-\bar{x}_{i}\right)^{2} ;
$$

the sample of $n$ observations has then been divided into $g$ groups, the $i$ th group containing $n_{i}$ observations with mean $\bar{x}, x_{i j}$ being the $j$ th observation in the $i$ group; c) one might continue this hierarchical process for a total of $n-1$ mergers, when there would be only one group which would encompass all the planetaries - (Ward 1963; Ward \& Hook 1963). In any real-life problem, the clusters so produced must, nevertheless, satisfy the criteria of compactness and isolation, that is, the groups must be well isolated from each other and, at the same time, the distance between the members of each group must be minimal. In other words, one seeks a partition that maximizes the between-class variance as well as minimizes the intra-class variance. This criterion, or dissimilarity, may be expressed as follows:

$$
V\left(P_{1}\right)-V\left(P_{2}\right)=V(g)-V\left(g_{a}\right)-V\left(g_{b}\right)
$$

where $V$ stands for variance, $P_{1}$ and $P_{2}$ are the previous and present partitions, respectively, where any original groups (classes) $g_{a}$ and $g_{b}$ have been agglomerated into a group $g$.

This technique has been applied to a sub-sample of 159 well-known PN using the PROC.-CLUSTERS.A.S. package facilities with the IBM 9221 computer of the Universidad de Santiago de Chile (USACH). This subsampling is to be regarded as having been done with no previous knowledge of any subdivisions whatsoever.

The reader may refer to Murtagh \& Heck (1987, pp 77-83) for references of works employing cluster analysis in Astronomy up to that year.

\subsection{The artificial neural network}

The ANN interests physicists, astronomers, engineers, computer and cognitive scientists, neuro-physiologists, biologists, and philosophers. The ANN can be characterized as computational tools with such properties as the ability to learn, generalize, and organize (classify) data, and of which operation is based on parallel processing (see Feldman \& Ballard 1982; McClelland \& Rumelhart 1986; Rumelhart \& McClelland 1986; Rumelhart et al. 1986; Josin 1988; Hertz et al. 1991; Kröse \& van der Smagt 1993, among others).

In Astronomy, the use of ANN increased recently in a significant manner, where most efforts have been centered on morphological classification of galaxies (Naim 1994; Adams \& Wooley 1994; Lahav et al. 1995), automated star/galaxy discrimination (Odewahn \& Nielsen 1994), spectral classification of galaxies (Sodré \& Cuevas 1994), stellar spectral classification (Storrie-Lombardi et al. 1994), and predictions of solar activity (Calvo et al. 1995), among others.

There are many learning methods for ANNs by now. The main categorization of these methods is by the learning process: it may be supervised or unsupervised. In the first case, the ANN is trained by providing it with both input and matching output patterns; these patterns are provided either by an external teacher (an expert in the particular problem, for instance) or by the system which contains the ANN (self-supervised). The second process, or self-organization, the ANN is autonomous in the sense that in this paradigm the system itself discovers statistically meaningful features of the input sample, thus developing its own representation of the input population.

In this work we have used the supervised learning package by Ripley (1994a, b), which consists in a feed-forward, back-propagation, algorithm with three free parameters: the weight decay, the hidden layer node number, and the number of subsidiary lines specifying the connection of one of the units to a second range ones in our adopted 
topologies (see Ripley 1992, 1993, 1994a, and 1994b for details). These parameters have been determined and crossvalidated as described in item 3 . The calculations have been performed with a Sun SPARCstation2 of the LNA.

\section{The results}

The cluster analysis produced a total of 6 groups, identified as follows: two groups correspond to Type I, and the other four to Types IIa, IIb, III, and IV, respectively. The subdivision of Type I PN is discussed in detail by Faúndez-Abans et al. (1995) and Ormeño et al. (1996). In this work we ignore this separation for the sake of comparison with the previous classification. The results are displayed in Table 1, where the different columns show: 1) the common name, 2) the galactic coordinates designation ("PN G", after Acker et al. 1992), 3) the previous classification made by other authors (FaúndezAbans \& Maciel 1986; 1988; de Freitas-Pacheco et al. 1991 and references quoted therein, Chiappini 1993; Maciel \& Chiappini 1994, "O"), 4) the classification by the cluster analysis ("CA"), 5) the classification by the ANN, and 6) our adopted type of PN ("A").

The maximum likelihood discriminant rule, applied to the five groups, shows the following coincidence percentages between the previous and the cluster division: $74 \%$ for Type I, $79.5 \%$ and $87.5 \%$ for Types IIa and IIb, respectively, $50 \%$ for Type III and $100 \%$ for Type IV. The poor result for Type III is explained by the non-inclusion of the peculiar radial velocity data (which, following Peimbert's works, is an important criterion of classification for Type III PN); the abundance parameters constitute a more homogeneous and free from large errors set.

In order to ascertain the ANN's learning ability, part of the coincident objects was used as a training set for the Ripley's ANN computer program. These nebulae have been chosen randomly among those with previously well-established PN type because of their previous wellestablished PN type; they represent roughly $81 \%$ of the whole sample. The training and test samples were composed by 126 and 63 independent nebulae, respectively ( $2 / 3$ and $1 / 3$ of the $81 \%$ sample above). The topologies employed were: 5 input units $(\mathrm{He}, \mathrm{O}, \mathrm{N}, \mathrm{S}$, and $\mathrm{Ar}$ abundance ratios), 5 output units, and tentatively six different widths for the hidden layer, namely, 3, 5, 10, 20, 25 , and 30 units. More than 20 hidden layer units did not improve significantly the errors, so we adopted the 5:20:5 topology to start with. In this topology, each unit of the hidden layer has five input connections, and each unit of the output layer has twenty input connections.

After having classified nebulae with these known five ratios, we classified those with missing $\mathrm{Ar}$ and then those missing both $\mathrm{Ar}$ and $\mathrm{S}$ ratios, employing 4:20:5 and 3:20:5 topologies, respectively.

The ANN was re-trained each time the number of objects increased due to the decrease in the number of
Table 1. The planetary nebulae sample

\begin{tabular}{|c|c|c|c|c|c|}
\hline Name & PN G & $\mathrm{O}$ & CA & ANN & A \\
\hline A $21(\mathrm{YM}-29)$ & $205.1+14.2$ & I & I & I & I \\
\hline $\mathrm{BD}+30.3639$ & $064.7+05.5$ & IIb & $\mathrm{IIb}$ & - & IIb \\
\hline BoBn-1 & $108.4-76.1$ & IV & IV & IV & IV \\
\hline Cn 1-5 & $002.2-09.4$ & - & - & IIa & IIa \\
\hline Cn 2-1 & $356.2-04.4$ & IIa & IIa & IIa & IIa \\
\hline Cn 3-1 & $038.2+12.0$ & III & III & - & III \\
\hline DdDm-1 & $061.9+41.3$ & IV & IV & IV & IV \\
\hline Fg 1 & $290.5+07.9$ & IIb & - & IIb & IIb \\
\hline H $1-17$ & $358.3+03.3$ & - & - & IIa & IIa \\
\hline H $1-18$ & $357.6+02.6$ & I & I & I & I \\
\hline H $1-20$ & $358.9+03.2$ & - & - & III-IV & III \\
\hline H $1-23$ & $357.6+01.7$ & I & I & I & I \\
\hline Н 1-27 & $005.0+04.4$ & - & - & III-IV & III \\
\hline Н $1-29$ & $355.2-02.5$ & - & - & IIb & IIb \\
\hline H 1-31 & $355.1-02.9$ & - & - & IIa-III & III \\
\hline H $1-35$ & $355.7-03.5$ & - & - & III & III \\
\hline H $1-5$ & $356.7-06.4$ & - & IIb & - & IIb \\
\hline H 1-54 & $002.1-04.2$ & III & IV & III & III \\
\hline H $1-5$ & $005.1-03.0$ & - & - & III & III \\
\hline H 1-59 & $003.8-04.3$ & I & IIa & I & I \\
\hline H 1-6 & 007.0 & - & - & IIa & IIa \\
\hline H 2-1 & $006.3+04.4$ & I & I & I & I \\
\hline H 2-46 & $000.8-07.6$ & - & - & IIb & IIb \\
\hline H 4-1 & $049.3+88.1$ & IV & IV & IV & IV \\
\hline $\mathrm{Hb} 04$ & $003.1+02.9$ & I & I & IIa & IIa \\
\hline $\mathrm{Hb} 05$ & $359.3-00.9$ & I & I & I & I \\
\hline $\mathrm{Hb} 06$ & $007.2+01.8$ & I & I & I & I \\
\hline b 12 & $111.8-02.8$ & IIb & III & IIb & IIb \\
\hline He 2-005 & $264.4-12.7$ & III & $\mathrm{IIb}$ & III-IV & III \\
\hline He 2-007 & $264.1-08.1$ & III & IIb & III & III \\
\hline He 2-009 & $258.1-00.3$ & III & III & III-IV & III \\
\hline $\mathrm{He}$ & 261.6 & I & I & I & I \\
\hline He $2-021$ & $275.3-04.7$ & IIb & III & III & IIb \\
\hline He $2-037$ & $274.6+03.5$ & IIa & - & IIa & IIa \\
\hline He $2-047$ & $285.6-02.7$ & - & - & IIa-III & IIa: \\
\hline He $2-048$ & $282.9+03.8$ & IIb & - & IIb & IIb \\
\hline He $2-051$ & $288.8-05.2$ & I & - & I & I \\
\hline He $2-055$ & $286.3+02.8$ & IIb & - & IIb & IIb \\
\hline He $2-071$ & $296.4-06.9$ & - & - & III & III \\
\hline He 2-086 & $300.7-0$ & I & I & I & I \\
\hline He 2-099 & $309.0-04.2$ & IIb & - & IIb & IIb \\
\hline He $2-108$ & $316.1+08.4$ & III & - & IIb-III & III \\
\hline He 2-111 & $15.0-00.3$ & I & I & I & I \\
\hline He $2-112$ & 319.2 & I & I & I & I \\
\hline He $2-115$ & $21.3+02.8$ & IIb & $\mathrm{IIb}$ & IIb & IIb \\
\hline He $2-117$ & $320.9+02.0$ & I & IIb & I-IIa & IIa \\
\hline He $2-118$ & $327.5+13.3$ & - & - & IIa & IIa \\
\hline He $2-119$ & 317.1 & I & - & I & I \\
\hline He $2-123$ & $23.9+02.4$ & IIa & I & I & I \\
\hline He 2-131 & $315.1-13.0$ & III & III & - & III \\
\hline He $2-140$ & $327.1-01.8$ & - & - & IIa & IIa \\
\hline He $2-141$ & $325.4-04.0$ & IIa & - & IIa & IIa \\
\hline He 2-149 & $329.4-02.7$ & - & - & III-IV & III \\
\hline He $2-153$ & $330.6-02.1$ & I & I & I & I \\
\hline He $2-157$ & $331.0-02.7$ & IIa & - & IIa & IIa \\
\hline He $2-158$ & $327.8-06.1$ & IIb & IIa & IIb-III & IIb \\
\hline He $2-164$ & $332.0-03.3$ & I & $\mathrm{IIb}$ & IIb & IIb \\
\hline He $2-170$ & $332.3-04.2$ & - & - & IIa-IIb & IIa: \\
\hline He $2-175$ & $345.6+06.7$ & - & - & I & I \\
\hline
\end{tabular}


Table 1. continued

\begin{tabular}{|c|c|c|c|c|c|}
\hline Name & $\overline{P N ~ G}$ & $\mathrm{O}$ & CA & $\overline{A N N}$ & $\mathrm{~A}$ \\
\hline He 2-406 & $008.6-07.0$ & - & - & $\mathrm{I}$ & I \\
\hline $\mathrm{Hu}$ 1-1 & $119.6-06.7$ & IIa & IIa & IIa & IIa \\
\hline $\mathrm{Hu} 1-2$ & $086.5-08.8$ & I & I & I & I \\
\hline $\mathrm{Hu} 2-1$ & $051.4+09.6$ & III & III & III & III \\
\hline IC 0351 & $159.0-15.1$ & $\mathrm{IIb}$ & $\mathrm{IIb}$ & IIb & $\mathrm{IIb}$ \\
\hline IC 0418 & $215.2-24.2$ & $\mathrm{IIb}$ & $\mathrm{IIb}$ & IIb & $\mathrm{IIb}$ \\
\hline IC 1297 & $358.3-21.6$ & IIa & IIa & IIa & IIa \\
\hline IC 1747 & $130.2+01.3$ & IIa & IIa & IIa & IIa \\
\hline IC 2003 & $161.2-14.8$ & IIa & IIa & IIa & IIa \\
\hline IC 2149 & $166.1+10.4$ & IIa & IIa & IIa & IIa \\
\hline IC 2165 & $221.3-12.3$ & IIa & IIa & IIa & IIa \\
\hline IC 2448 & $285.7-14.9$ & IIa & - & IIa & IIa \\
\hline IC 2501 & $281.0-05.6$ & IIa & IIa & IIa & IIa \\
\hline IC 2621 & $291.6-04.8$ & IIa & IIa & IIa & IIa \\
\hline IC 3568 & $123.6+34.5$ & $\mathrm{IIb}$ & IIb & IIb & $\mathrm{IIb}$ \\
\hline IC 4406 & $319.6+15.7$ & I & I & I & I \\
\hline IC 4593 & $025.3+40.8$ & III & IIa & III & III \\
\hline IC 4634 & $000.3+12.2$ & III & III & III & III \\
\hline IC 4673 & $003.5-02.4$ & I & IIa & I & I \\
\hline IC 4732 & $010.7-06.4$ & III & III & III & III \\
\hline IC 4776 & $002.0-13.4$ & $\mathrm{IIb}$ & IIb & IIb & $\mathrm{IIb}$ \\
\hline IC 4846 & $027.6-09.6$ & III & III & III & III \\
\hline IC 4997 & $058.3-10.9$ & III & - & III & III \\
\hline IC 5117 & $089.8-05.1$ & IIa & IIb & IIa & IIa \\
\hline IC 5217 & $100.6-05.4$ & IIa & IIa & IIa & IIa \\
\hline J 320 & $190.3-17.7$ & $\mathrm{IIb}$ & IIb & IIb & $\mathrm{IIb}$ \\
\hline J 900 & $194.2+02.5$ & $\mathrm{IIb}$ & IIb & IIb & $\mathrm{IIb}$ \\
\hline K 3-61 & $096.3+02.3$ & I & $\mathrm{I}$ & I & I \\
\hline K 3-67 & $165.5-06.5$ & III & IIb & IIa & IIa \\
\hline M 1-01 & $130.3-11.7$ & IIb & III & IIb & IIb \\
\hline M 1-04 & $147.4-02.3$ & $\mathrm{IIb}$ & I & IIb & $\mathrm{IIb}$ \\
\hline M 1-05 & $184.0-02.1$ & $\mathrm{IIb}$ & IIb & IIb & $\mathrm{IIb}$ \\
\hline M 1-06 & $211.2-03.5$ & - & - & III & III \\
\hline M 1-08 & $210.3+01.9$ & I & - & IIa & IIa \\
\hline M 1-12 & $235.3-03.9$ & - & - & IV & IV \\
\hline M 1-13 & $232.4-01.8$ & I & IIa & I & I \\
\hline M 1-17 & $228.8+05.3$ & I & I & IIa & IIa \\
\hline M 1-22 & $007.5+07.4$ & - & - & I & I \\
\hline M 1-25 & $004.9+04.9$ & IIa & IIa & IIa & IIa \\
\hline M 1-26 & $358.9-00.7$ & III & III & III & III \\
\hline M 1-30 & $355.9-04.2$ & - & - & IIa & IIa \\
\hline M 1-34 & $357.9-05.1$ & IIa & IIa & I & I \\
\hline M 1-35 & $003.9-02.3$ & I & I & I & I \\
\hline M 1-40 & $008.3-01.1$ & I & I & I & I \\
\hline M 1-42 & $002.7-04.8$ & $\mathrm{I}$ & I & I & I \\
\hline M 1-50 & $014.6-04.3$ & $\mathrm{IIb}$ & $\mathrm{IIb}$ & IIb & $\mathrm{IIb}$ \\
\hline M 1-51 & $020.9-01.1$ & I & IIa & IIa & IIa \\
\hline M 1-54 & $016.0-04.3$ & IIa & IIa & I & I \\
\hline M 1-56 & $016.1-04.7$ & - & - & IIa-III & III \\
\hline M 1-57 & $022.1-02.4$ & IIa & I & IIa-III & IIa \\
\hline M 1-60 & $019.7-04.5$ & - & - & IIa & IIa \\
\hline M 1-61 & $019.4-05.3$ & 一 & - & IIb & $\mathrm{IIb}$ \\
\hline M 1-72 & $054.4-02.5$ & - & - & III & III \\
\hline M 1-74 & $052.2-04.0$ & IIa & IIa & IIa & IIa \\
\hline M 1-75 & $068.8-00.0$ & I & $\mathrm{I}$ & I & I \\
\hline M 1-78 & $093.5+01.4$ & III & III & III & III \\
\hline M 1-80 & $107.7-02.2$ & IIa & IIa & IIa & IIa \\
\hline M 2-02 & $147.8+04.1$ & IIb & - & IIb & IIb \\
\hline M 2-06 & $353.3+06.3$ & III & III & III & III \\
\hline
\end{tabular}

Table 1. continued

\begin{tabular}{|c|c|c|c|c|c|}
\hline Name & $P N$ G & $\mathrm{O}$ & CA & ANN & $\mathrm{A}$ \\
\hline $\bar{M} 2-09$ & $010.8+18.0$ & III & IV & III & III \\
\hline M 2-10 & $354.2+04.3$ & IIa & IIa & IIa-III & IIa \\
\hline M 2-11 & $356.9+04.5$ & - & - & IIb & IIb \\
\hline M 2-13 & $011.1+11.5$ & - & - & IIa-III & IIa: \\
\hline M 2-18 & $357.4-03.5$ & - & - & $\mathrm{IIb}$ & IIb \\
\hline M 2-23 & $002.2-02.7$ & III & $\mathrm{IIb}$ & III & III \\
\hline M 2-29 & $004.0-03.0$ & - & - & III-IV & IV \\
\hline M 2-30 & $003.7-04.6$ & - & - & III & III \\
\hline M 2-42 & $008.2-04.8$ & - & - & IIa-III & III \\
\hline M 2-46 & $024.8-02.7$ & - & - & IIa & IIa \\
\hline M 2-50 & $097.6-02.4$ & III & III & III & III \\
\hline M 3-01 & $242.6-11.6$ & III & - & III & III \\
\hline M 3-02 & $240.3-07.6$ & I & IIa & III & III \\
\hline M 3-03 & $221.7+05.3$ & I & - & I & I \\
\hline M 3-04 & $241.0+02.3$ & IIa & - & IIa & IIa \\
\hline M 3-05 & $245.4+01.6$ & I & - & IIa & IIa \\
\hline M 3-06 & $253.9+05.7$ & $\mathrm{IIb}$ & $\mathrm{IIb}$ & $\mathrm{IIb}$ & $\mathrm{IIb}$ \\
\hline M 3-10 & $358.2+03.6$ & - & - & IIa & IIa \\
\hline M 3-14 & $355.4-02.4$ & I & I & I & I \\
\hline M 3-15 & $006.8+04.1$ & IIa & IIa & IIa & IIa \\
\hline M 3-21 & $355.1-06.9$ & - & - & IIa & IIa \\
\hline M 3-29 & $004.0-11.1$ & III & $\mathrm{IIb}$ & IIa & IIa \\
\hline M 3-38 & $356.9+04.4$ & - & - & IIa-III & III \\
\hline M 4-3 & $357.2+07.4$ & III & IIb & III & III \\
\hline Me 1-1 & $052.5-02.9$ & I & I & I & I \\
\hline Me 2-1 & $342.1+27.5$ & III & IIb & III & III \\
\hline Me 2-2 & $100.0-08.7$ & $\mathrm{I}$ & IIa & I & I \\
\hline $\mathrm{Mz} 2$ & $329.3-02.8$ & I & I & I & I \\
\hline Mz 3 & $331.7-01.0$ & I & I & I & I \\
\hline NGC 0650-51 & $130.9-10.5$ & I & I & I & I \\
\hline NGC 1535 & $206.4-40.5$ & IIb & $\mathrm{IIb}$ & $\mathrm{IIb}$ & $\mathrm{IIb}$ \\
\hline NGC 2022 & $196.6-10.9$ & $\mathrm{IIb}$ & $\mathrm{IIb}$ & $\mathrm{IIb}$ & IIb \\
\hline NGC 2242 & $170.3+15.8$ & III & - & III-IV & III \\
\hline NGC 2346 & $215.6+03.6$ & I & IIa & IIa & IIa \\
\hline NGC 2371-72 & $189.1+19.8$ & IIa & III & IIa & IIa \\
\hline NGC 2392 & $197.8+17.3$ & IIa & IIa & IIa & IIa \\
\hline NGC 2438 & $231.8+04.1$ & IIa & IIa & IIa & IIa \\
\hline NGC 2440 & $234.8+02.4$ & I & I & $\mathrm{I}$ & I \\
\hline NGC 2452 & $243.3-01.0$ & I & I & I & I \\
\hline NGC 2610 & $239.6+13.9$ & III & - & III & III \\
\hline NGC 2792 & $265.7+04.1$ & IIa & - & $\mathrm{I}$ & $\mathrm{I}$ \\
\hline NGC 2818 & $261.9+08.5$ & I & IIa & I & I \\
\hline NGC 2867 & $278.1-05.9$ & IIa & IIa & IIa & IIa \\
\hline NGC 2899 & $277.1-03.8$ & I & - & I & I \\
\hline NGC 3132 & $272.1+12.3$ & I & - & IIa & IIa \\
\hline NGC 3195 & $296.6-20.0$ & IIa & - & IIa & IIa \\
\hline NGC 3211 & $286.3-04.8$ & $\mathrm{IIb}$ & $\mathrm{IIb}$ & $\mathrm{IIb}$ & IIb \\
\hline NGC 3242 & $261.0+32.0$ & IIb & IIb & $\mathrm{IIb}$ & IIb \\
\hline NGC 3587 & $148.4+57.0$ & IIa & - & IIa & IIa \\
\hline NGC 3918 & $294.6+04.7$ & IIa & IIa & IIa & IIa \\
\hline NGC 4361 & $294.1+43.6$ & III & III & III & III \\
\hline NGC 5189 & $307.2-03.4$ & I & I & I & I \\
\hline NGC 5307 & $312.3+10.5$ & IIb & III & $\mathrm{IIb}$ & IIb \\
\hline NGC 5315 & $309.1-04.3$ & I & $\mathrm{I}$ & $\mathrm{I}$ & I \\
\hline NGC 5873 & $331.3+16.8$ & III & - & III & III \\
\hline NGC 5882 & $327.8+10.0$ & IIa & IIa & IIa & IIa \\
\hline NGC 5979 & $322.5-05.2$ & III & - & IIb & IIb \\
\hline NGC 6153 & $341.8+05.4$ & I & I & I & I \\
\hline NGC 6210 & $043.1+37.7$ & IIb & IIb & $\mathrm{IIb}$ & IIb \\
\hline
\end{tabular}


Table 1. continued

\begin{tabular}{|c|c|c|c|c|c|}
\hline Name & PN G & $\mathrm{O}$ & $\mathrm{CA}$ & ANN & $\mathrm{A}$ \\
\hline$\overline{\text { NGC } 6302}$ & $349.5+01.0$ & $\mathrm{I}$ & I & $\mathrm{I}$ & $\mathrm{I}$ \\
\hline NGC 6309 & $009.6+14.8$ & IIa & IIa & IIa & IIa \\
\hline NGC 6369 & $002.4+05.8$ & $\mathrm{I}$ & $\mathrm{IIb}$ & IIa-III & III \\
\hline NGC 6439 & $011.0+05.8$ & IIa & IIb & IIa & IIa \\
\hline NGC 6445 & $008.0+03.9$ & $\mathrm{I}$ & $\mathrm{I}$ & $\mathrm{I}$ & I \\
\hline NGC 6537 & $010.1+00.7$ & I & - & I & I \\
\hline NGC 6543 & $096.4+29.9$ & $\mathrm{IIb}$ & $\mathrm{IIb}$ & IIb & $\mathrm{IIb}$ \\
\hline NGC 6563 & $358.5-07.3$ & IIa & $\mathrm{IIb}$ & IIa & IIa \\
\hline NGC 6565 & $003.5-04.6$ & IIa & IIa & IIa & IIa \\
\hline NGC 6567 & $011.7-00.6$ & $\mathrm{IIb}$ & III & IIb-III & III \\
\hline NGC 6572 & $034.6+11.8$ & IIa & IIa & IIa & IIa \\
\hline NGC 6578 & $010.8-01.8$ & IIa & $\mathrm{IIb}$ & IIa & IIa \\
\hline NGC 6620 & $005.8-06.1$ & I & IIa & $\mathrm{I}$ & I \\
\hline NGC 6629 & $009.4-05.0$ & IIb & $\mathrm{IIb}$ & $\mathrm{IIb}$ & IIb \\
\hline NGC 6644 & $008.3-07.3$ & III & $\mathrm{IIb}$ & III & III \\
\hline NGC 6720 & $063.1+13.9$ & IIa & IIa & IIa & IIa \\
\hline NGC 6741 & $033.8-02.6$ & I & $\mathrm{I}$ & $\mathrm{I}$ & I \\
\hline NGC 6751 & $029.2-05.9$ & I & I & I & I \\
\hline NGC 6778 & $034.5-06.7$ & I & I & I & I \\
\hline NGC 6781 & $041.8-02.9$ & $\mathrm{I}$ & $\mathrm{I}$ & $\mathrm{I}$ & I \\
\hline NGC 6790 & $037.8-06.3$ & $\mathrm{IIb}$ & $\mathrm{IIb}$ & $\mathrm{IIb}$ & $\mathrm{IIb}$ \\
\hline NGC 6803 & $046.4-04.1$ & I & I & I & I \\
\hline NGC 6807 & $042.9-06.9$ & III & $\mathrm{IIb}$ & III & III \\
\hline NGC 6818 & $025.8-17.9$ & IIa & $\mathrm{IIb}$ & IIa & IIa \\
\hline NGC 6826 & $083.5+12.7$ & $\mathrm{IIb}$ & $\mathrm{IIb}$ & $\mathrm{IIb}$ & $\mathrm{IIb}$ \\
\hline NGC 6833 & $082.5+11.3$ & III & $\mathrm{IIb}$ & III & III \\
\hline NGC 6853 & $060.8-03.6$ & $\mathrm{I}$ & IIa & $\mathrm{I}$ & I \\
\hline NGC 6879 & $057.2-08.9$ & $\mathrm{IIb}$ & $\mathrm{IIb}$ & $\mathrm{IIb}$ & IIb \\
\hline NGC 6881 & $074.5+02.1$ & I & $\mathrm{I}$ & $\mathrm{I}$ & I \\
\hline NGC 6884 & $082.1+07.0$ & $\mathrm{IIb}$ & $\mathrm{IIb}$ & $\mathrm{IIb}$ & IIb \\
\hline NGC 6886 & $060.1-07.7$ & IIa & IIa & IIa & IIa \\
\hline NGC 6891 & $054.1-12.1$ & IIb & IV & $\mathrm{IIb}$ & IIb \\
\hline NGC 6894 & $069.4-02.6$ & IIa & - & IIa & IIa \\
\hline NGC 6905 & $061.4-09.5$ & IIa & IIa & IIa & IIa \\
\hline NGC 7008 & $093.4+05.4$ & I & - & $\mathrm{I}$ & I \\
\hline NGC 7009 & $037.7-34.5$ & IIa & IIa & IIa & IIa \\
\hline NGC 7026 & $089.0+00.3$ & IIa & IIa & IIa & IIa \\
\hline NGC 7027 & $084.9-03.4$ & IIa & IIa & IIa & IIa \\
\hline NGC 7293 & $036.1-57.1$ & $\mathrm{I}$ & - & I & I \\
\hline NGC 7354 & $107.8+02.3$ & I & I & $\mathrm{I}$ & I \\
\hline NGC 7662 & $106.5-17.6$ & IIb & $\mathrm{IIb}$ & $\mathrm{IIb}$ & IIb \\
\hline PB 6 & $278.8+04.9$ & $\mathrm{I}$ & $\mathrm{I}$ & $\mathrm{I}$ & I \\
\hline PC 14 & $336.2-06.9$ & IIa & - & IIa & IIa \\
\hline Pe 1-01 & $285.4+01.5$ & - & - & IIa & IIa \\
\hline Pe 1-17 & $024.3-03.3$ & I & IIa & I & I \\
\hline Pe $1-18$ & $027.3-02.1$ & IIa & I & IIa-III & IIa \\
\hline Pe 2-14 & $013.0-04.3$ & - & - & IV & IV:: \\
\hline Ps-1(K 648) & $065.0-27.3$ & IV & IV & IV & IV \\
\hline Sn-1 & $013.3+32.7$ & III & $\mathrm{IIb}$ & III & III \\
\hline SwSt-1 & $001.5-06.7$ & III & $\mathrm{IIb}$ & III & III \\
\hline Tc-1 & $345.2-08.8$ & III & - & III & III \\
\hline Th 2-A & $306.4-00.6$ & IIa & - & IIa & IIa \\
\hline Vd 1-1 & $344.2+04.7$ & - & - & IV & IV \\
\hline Vy 1-2 & $053.3+24.0$ & III & $\mathrm{IIb}$ & III & III \\
\hline Vy 2-1 & $007.0-06.8$ & - & - & IIb-III & III \\
\hline Vy $2-2$ & $045.4-02.7$ & III & III & III & III \\
\hline
\end{tabular}

parameters, being forced to recognize the new sample once again. This ensured the self-consistency of the classification. Even then, for 29 objects (Type III included) the ANN do not show the best agreement and display indecisions between IIa-III, IIb-III, and III-IV; for 6 objects it assigns similar probabilities for I-IIa and IIa-IIb. In this step we used the radial velocities compiled by Acker et al. (1992) to improve the results. We trained the ANN with 138 objects using $\mathrm{He}, \mathrm{O}, \mathrm{N}$, and the radial velocity (topology 4:20:5), and again forced to recognize the whole sample (203 objects with velocities). The before precisely classified objects matched this last test; the 29 objects became 20 , and the 6,2 . The errors we have obtained for both training and test stages are of $1 \%$ and $2 \%$ for the 5:20:5 and 4:20:5 topologies, respectively. For the topology $3: 20: 5$, they are close to $2 \%$ and $7 \%$, respectively. In spite of this error value, 3 seems to be the minimum number of input parameters for this ANN classifier to yield satisfactory results.

\section{Discussion}

In the beginning of this work, the assumption was made that the sample is homogeneous and there was no previous knowledge of any classification of the PN.

The cluster analysis has proved to be suitable as a first guess of classification, and the coincidence with the previous classification was $70.3 \%$.

The neural network was trained with $2 / 3$ of these coincident objects (topology 5:20:5) in the first run and forced to recognize the whole sample. Based on these results we have increased the number of training objects for the subsequent topologies. In the final ANN classification, $74.1 \%$ of the objects match the previous classification, and $13.2 \%$ the ANN "indecisions" (two most probable types) include the previous type. The inclusion of the radial velocity values did not improve significantly the performance of the ANN in the case of Type III objects; furthermore, the velocity parameter has a broad scatter which is reflected in the ANN error. We have adopted basically the classification given by the ANN. In four cases, when the He abundance is missing, we have adopted the classification of the cluster analysis.

The comparison between the results of the cluster analysis and the ANN's shows $64.5 \%$ of coincidence in their classification.

The ANN became an interesting tool of classification, principally because it can be trained to a degree that warrants satisfactorily reliable results without loss of generalization ability. In this work, $41 \mathrm{PN}$ have been classified for the first time, and $13 \mathrm{PN}$ had their types changed by the ANN.

Obviously, a few PN of the sample may suffer changes in classification in the future mainly because the abundance values may change due to electron temperature fluctuations (see Dinerstein et al. 1985; Peimbert et al. 1993). 
But, in general, it is expected that the present classification remains.

With reference to the previous criteria of classification by other authors, the grouping of our sample does not agree strictly for each parameter criterion. For example, for Type I PN the $\mathrm{He} / \mathrm{H}$ ratio spreads from 0.090 to 0.186 in our sample, where the first value is lower than the $\mathrm{He} / \mathrm{H}$ cut-off criterion for the Type I PN ( $\gtrsim 0.125)$. Also, we have classified a few originally Type I objects as IIa, IIb, and III, which have a high He abundance value for this class if one follows strictly the criterion. Another example is Type III PN, where in our sample the radial velocity spreads from 5 to approximately $224 \mathrm{~km} / \mathrm{s}$. The abundance parameters of these objects in several cases are close to the IIa, IIb, and IV ones; anyway, the cluster analysis and the ANN can group partially this class using only abundance parameters. We expect that an ANN with more than five abundance parameters classifies the Type III better.

The four well-known Type IV PN BoBn-1, DdDm$1, \mathrm{H} 4-1$, and Ps-1, have been recognized correctly by the ANN, and four more ones have been added to the list, namely, M 1-12, M 2-29, Pe 2-14, and Vd 1-1. There is not, nonetheless, much information on these objects in the literature. M 1-12 has had its distance individually determined by Zhang \& Kwok (1993), a statistical one, of 6.42 kpc, given by van de Steene \& Zijlstra (1994) and Zhang (1995); and $6.48 \mathrm{kpc}$ for the final distance scale for the galactic bulge $\mathrm{PN}$ ), and no radial velocity; M 2-29 shows a high radial velocity $114 \pm 11 \mathrm{~km} / \mathrm{s}$, given by Schneider et al. (1983), and has been quoted as a galactic halo object by Peimbert (1991), of which distance runs from $9.31 \mathrm{kpc}$ to $11.33 \mathrm{kpc}$ (van de Steene \& Zijlstra 1994) and Cahn et al. (1992), respectively; Zhang (1995) calculates a statistical distance of $9.31 \mathrm{kpc}$ and a final distance for the galactic bulge $\mathrm{PN}$ of $\sim 9.98 \mathrm{kpc}$. Pe $2-14$ has neither individual distance nor radial velocity determinations, just a statistical estimate of the distance ranging from $3.1 \mathrm{kpc}$ to $6.07 \mathrm{kpc}$, as given by Maciel (1984) and Cahn \& Kaler (1971); finally, Vd 1-1 has no distance determinations and its high radial velocity is $-142.1 \pm 3.5 \mathrm{~km} / \mathrm{s}$ (Schneider et al. 1983). Further observational studies of these objects will be made in the near future.

Either the cluster analysis or the supervised ANN seems to be a suitable tool for the problem of $\mathrm{PN}$ classification. The results obtained in Table 1 show that the parameters used "tell classes apart" and can be restricted to about 3 for a minimum reliable classification. When 5 input parameters are used, the ANN classification error improves notoriously, no matter what guesses on the weight decay are given because different combinations of these two variables yield results that are statistically equivalent. We expect that the application of both tools to Amnuel's data would yield the same results or, at least, complement them.
For the sake of illustration, Figs. 1-3 show the coincident objects as classified by Amnuel and by us in diagrams of $\mathrm{He}$ against $\mathrm{N}$ for low (L), intermediate (In), and massive (M) planetaries, respectively. A few of Amnuel's anomalous PN have been included in these samples. It is noted that Peimbert's types are intermixed on this scenario, as can be seen from these figures. It is only in Fig. 2 that a clear correlation appears for Class In PN. On the other hand, a more careful look at Fig. 1 makes it apparent that IIa and IIb nebulae seem to exhibit rather definite trends, with negative and positive slopes, respectively (excluding the deviant IIa M1-30). Here we pose the following question: if the $\mathrm{CN}$-cycle is important in all progenitor stars, and the ON-cycle plays a rôle in stars with $M \lesssim 3 M_{\odot}$ (Amnuel 1993), why would low-mass PN distribute themselves as in Fig. 1? An enhancement of $\mathrm{He}$ would be expected due to the $\mathrm{CN}$-cycle and the depletion of the initial $\mathrm{O}$ by the ON-cycle, being converted into secondary $\mathrm{N}$; both processes should possibly cause the trend seen in Fig. 2.

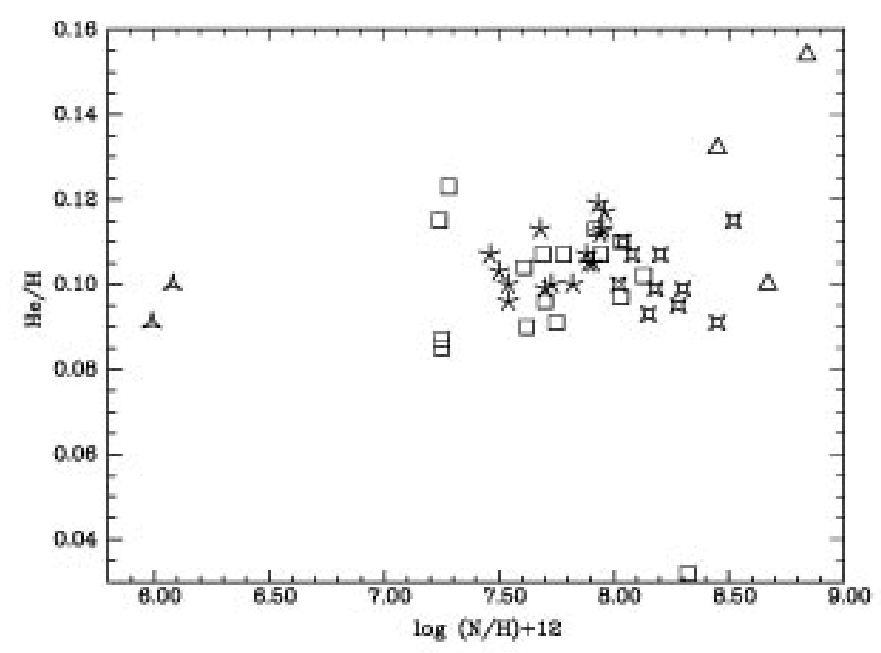

Fig. 1. Distribution of low-mass PN (L). The symbols represent Peimbert's types I $(\triangle)$, IIa (open cross), IIb (squeletal pentagon), III ( $\square)$, and IV (squeletal triangles)

We finally stress that the number of useful parameters in $\mathrm{PN}$ is restricted because of the lack of complete abundance data for all the PN known to present date. In a future work, we intend to employ an unsupervised ANN architecture, which is not very complex due to the low number of input parameters, as shown by the present work, and add the morphology as another input parameter.

Acknowledgements. This work was partially supported by CNPq (Brazil). One of us, M. I. O. A., thanks Claudio Silva for some help and comments on the cluster analysis and the Departamento de Física of the USACH (Chile) for partial support. We thank Laerte Sodré for kindly supplying the ANN program, for his help and advice on the use of the neural network, 


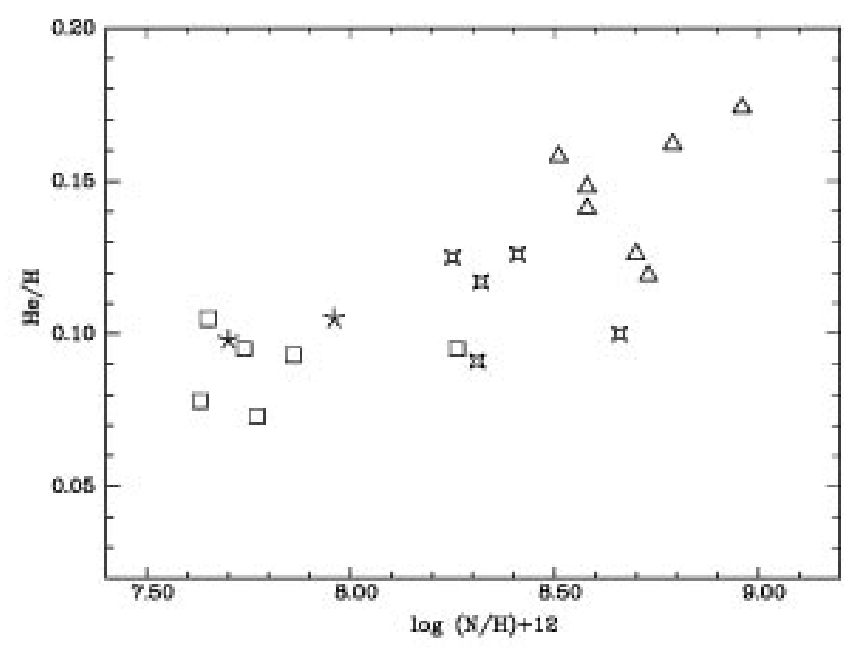

Fig. 2. The same as Fig. 1 for intermediate-mass planetaries (In)

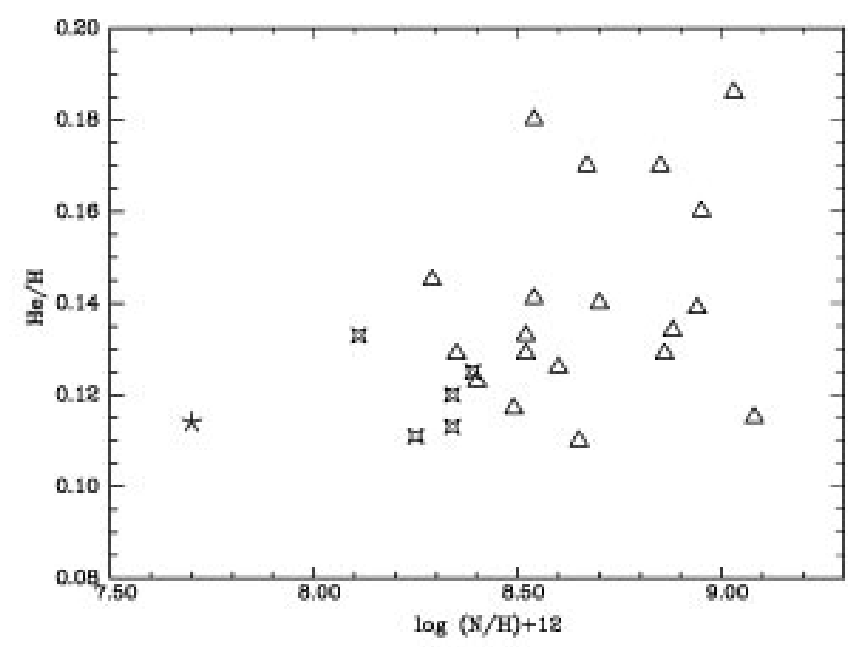

Fig. 3. The same as Fig. 1 for massive nebulae (M)

and reading of the manuscript. The neural network program is available via anonymous ftp from markov.stats.ox.ac.uk.

\section{References}

Acker A., Ochsenbein F., Stenholm B., Tylenda R., Marcout J., Schohn C., 1992, Strasbourg-ESO Catalogue of Galactic Planetary Nebulae, ESO, Munich

Adams A., Wooley A., 1994, Vistas Astron. 38, 273

Amnuel P.R., 1993, MNRAS 261, 263

Amnuel P.R., Guseinov, O.H., Rustamov, Yu.S., 1989, Ap\&SS 154,21

Anderberg M.R., 1973, Cluster Analysis for Applications. Academic Press, New York

Cahn J.H., Kaler J.B., 1971, ApJS 22, 319

Cahn J.H., Kaler J.B., Stanghellini L., 1992, A\&AS 94, 399

Calvo R.A., Ceccatto H.A., Piacentini R.D., 1995, ApJ 444, 916
Chiappini C., 1993, thesis, Evolução Química da Galáxia: a Razão de Enriquecimento entre O, ${ }^{4} \mathrm{He}$ e os Elementos Pesados, Universidade de São Paulo, Brazil

de Freitas-Pacheco J.A., Maciel W.J., Costa R.D.D., Barbuy B., 1991, A\&A 250, 159

Dinerstein H.L., Lester D.F., Werner M.W., 1985, ApJ 291, 561

Faúndez-Abans M., Maciel W.J., 1986, A\&A 158, 228

Faúndez-Abans M., Maciel W.J., 1987a A\&A 183, 324

Faúndez-Abans M., Maciel W.J., 1987b, Ap\&SS 129, 353

Faúndez-Abans M., Maciel W.J., 1988, Rev. Mex. Astron. Astrofís. 16, 105

Faúndez-Abans M., de Oliveira-Abans M., Ormeño M.I., 1995, A\&A (accepted for publication)

Feldman J.A., Ballard D.H., 1982, Cognitive Sci. 6, 205

Hertz J.A., Krogh A., Palmer R.G. 1991, Introduction to the Theory of Neural Computation, Addison-Wesley, Redwood City

Johnson R.A., Wichern D., 1986, in: Multivariate Analysis, Encyclopaedia Stat. Sci. 6. John Wiley \& Sons, New York

Josin G., 1988, Biolog. Cybernet. 59, 283

Kendall M.G., Stuart A., 1968, The Advanced Theory of Statistics, Vol. 3, Griffin, London

Köppen J., Acker A., Stenholm B., 1991, A\&A 248, 197

Kröse B.J.A., van der Smagt P.P., 1993, An Introduction to Neural Networks, Fifth Edition, University of Amsterdam

Lahav O., Naim A., Buta R.J., et al., 1995, Sci 267, 859

Maciel W.J., 1984, A\&AS 55, 253

Maciel W.J., 1989, Galactic Distribution, Radial Velocities, and Masses of PN. In: Torres-Peimbert S. (ed.) Proc. IAU Symp. 131, Planetary Nebulae. Kluwer Acad. Publ., Dordrecht, p. 73

Maciel W.J., Chiappini C., 1994, Ap\&SS 219, 231

Maciel W.J., Köppen J., 1994, A\&A 282, 436

Marriolt F.H.C., 1974, The Interpretation of Multiple Observations. Academic Press, New York

McClelland J.L., Rumelhart D.E., 1986, Parallel Distributed Processing: Explorations in the Microstructure of Cognition Vol. 2, The MIT Press

Murtagh F., Heck A., 1987, Multivariate Data Analysis, Ap\&SS Library. D. Reidel, Dordrecht

Naim A., 1994, Vistas Astron. 38, 265

Odewahn S.C., Nielsen M.L., 1994, Vistas Astron. 38, 281

Ormeño M.I., Faúndez-Abans M., de Oliveira-Abans M., 1996 (in preparation)

Peimbert M., 1978, Chemical Abundances in Planetary Nebulae. In: Terzian Y. (ed.) Proc. IAU Symp. 76, Planetary Nebulae - Observations and Theory. Reidel, Dordrecht, p. 215

Peimbert M., 1990, Rep. Prog. Phys. 53, 1559

Peimbert M., 1991, in: Terlevich R. et al. (eds.) Elements and the Cosmos. Cambridge Univ. Press

Peimbert M., Serrano A., 1980, Rev. Mex. Astron. Astrofís. 5,9

Peimbert M., Torres-Peimbert S., 1983, Type I Planetary Nebulae. In: Flower D.R. (ed.) Proc. IAU Symp. 103, Planetary Nebulae. Reidel, Dordrecht, p. 233

Peimbert M., Storey P.J., Torres-Peimbert S., 1993, ApJ 414, 626 
Ripley B.D., 1992, Software for Neural Networks Classification (private communication)

Ripley B.D., 1993, Statistical Aspects of Neural Networks. In: Barndorff-Nielsen O.E., Cox P.R., Jensen J.L. and Kenpall W.S. (eds.) Chaos and Networks - Statistical and Probabilistic Aspects. Chapman \& Hall London

Ripley B.D., 1994a, Neural Networks and Related Methods for Classification (with discussion), JRSSB 56, 409

Ripley B.D., 1994b, Neural Networks and Flexible Regression and Discrimination. In: Mardia K.V. (ed.) Advances in Applied Statistics 2, Statistics and Images. Carfax, Abingdon, pp. 39-57,

Rumelhart D.E., McClelland J.L., 1986, Parallel Distributed Processing: Explorations in the Microstructure of Cognition Vol. 1, The MIT Press

Rumelhart D.E., Hilton G.E., Williams R.J., 1986, Nat 323, 533
Schneider S.E., Terzian Y., Purgathofer A., Perinotto M., 1983, ApJS 52, 399

Sodré Jr.L., Cuevas H., 1994, Vistas Astron. 38, 287

Storrie-Lombardi M.C., Irwin M.J., von Hippel T., StorrieLombardi L.J., 1994, Vistas Astron. 38, 331

Tatsuoka M.M., 1971, Multivariate Analysis. John Wiley \& Sons, New York

Ward Jr.J.H., 1963, Hierarchical Grouping to Optimize on Objective Function, Statist. Assoc. 58, 236

Ward Jr.J.H., Hook M.E., 1963, Application of a Hierarchical Grouping Procedure to a Problem of Grouping Profiles, ed. Psychol. Measurement 23, 69

Van de Steene G.C., Zijlstra A.A., 1994, A\&AS 108, 485

Wishart D., 1969, Biometrics 22, 165

Zhang C.Y., 1995, ApJS 98, 659

Zhang C.Y., Kwok S., 1993, ApJS 88, 137 